(c) Verlag Chemie GmbH, D-6940 Weinheim, 1981 - Printed in the Federal Republic of Germany.

Verantwortlich für den Inhalt: Prof. Dr. Hans Musso, Karlsruhe. Redaktion: Dr. Hermann Zahn, München.

Anzeigenleitung: R. J. Roth, Weinheim.

Verlag Chemie GmbH (Geschäftsführer Dr. Helmut Grünewald und Hans Schermer), Pappelallec 3, Postfach 1260/1280, D-6940 Weinheim.

Die Wiedergabe von Gebrauchsnamen, Handelsnamen, Warenbezeichnungen und dgl. in dieser Zeitschrift berechtigt nicht zu der Annahme, daß solche Namen ohne weiteres von jedermann benutzt werden dürfen. Vielmehr handelt es sich häufig um gesetzlich geschützte eingetragene Warenzeichen, auch wenn sie nicht als solche gekennzeichnet sind.

Alle Rechte, insbesondere die der Übersetzung in fremde Sprachen, vorbehalten. Kein Teil dieser Zeitschrift darf ohne schriftliche Genehmigung des Verlages in irgendeiner Form - durch Photokopie, Mikrofilm oder irgendein anderes Verfahren - reproduziert oder in eine von Maschinen, insbesondere von Datenverarbeitungsmaschinen verwendbare Sprache überıragen oder übersetzt werden. - All rights reserved (including those of translation into foreign languages). No part of this issue may be reproduced in any form - by photoprint, microfilm, or any other means - nor transmitted or translated into a machine language without the permission in writing of the publishers. - Von einzelnen Beiträgen oder Teilen von ihnen dürfen nur einzelne Vervielfältigungsstücke für den persönlichen oder sonstigen eigenen Gebrauch hergestellt werden. Jede im Bereich eines gewerblichen Unternehmens hergestellte oder benutzte Kopie dient gewerblichen Zwecken gem. § 54(2) UrhG und verpflichtet zur Gebührenzahlung an die VG WORT, Abteilung Wissenschaft, Goethestr. 49,8000 München 2, von der die einzelnen Zahlungsmodalitäten zu erfragen sind. Die Weitergabe von Vervielfältigungen, gleichgültig zu welchem Zweck sie hergestellt werden, ist eine Urheberrechtsverletzung. Valid for users in the USA: The appearance of the code at the bottom of the first page of an article in this journal (serial) indicates the copyright owner's consent that copies of the article may be made for personal or internal use, or for the personal or internal use of specific clients. This consent is given on the condition, however, that the copier pay the stated percopy fee through the Copyright Clearance Center, Inc., for copying beyond that permitted by Sections 107 or 108 of the U.S. Copyright Law. This consent does not extend to other kinds of copying, such as copying for general distribution, for advertising or promotional purposes, for creating new collective work, or for resale. For copying from back volumes of this journal see »Permissions to Photo-Copy: Publisher's Fee List" of the CCC.

In der Zeitschrift werden keine Rezensionen veröffentlicht; zur Besprechung eingehende Bücher werden nicht zurückgesandt. Herstellung: Krebs-Gehlen Druckerei, Hemsbach (Bergstraße). 


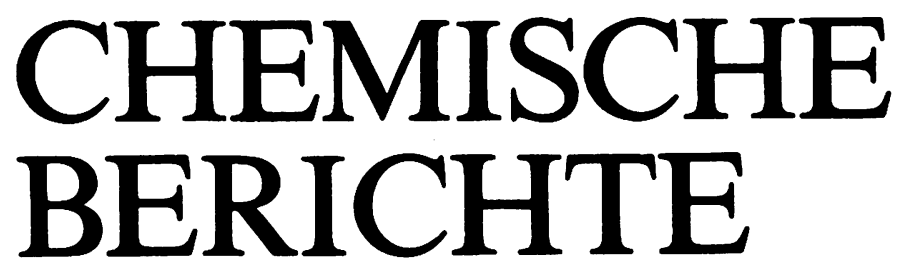

GEGRÜNDET 1868

114. JAHRGANG

HERAUSGEGEBEN IM AUFTRAG DER

GESELLSCHAFT DEUTSCHER CHEMIKER

VON

K. HAFNER - W. KIRMSE - H. MUSSO - H. NÖTH -

J. SAUER - E. WINTERFELDT

UNTER MITWIRKUNG VON

H. A. BRUNE - W. LÜTTKE - G. SPITELLER

REDAKTION: H. ZAHN

mit H. SCHILL, J. STREHLOW und A. WIELAND

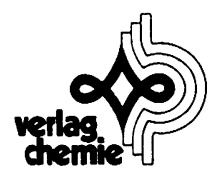




\section{BEMERKUNGEN DER REDAKTION}

1. Die Chemischen Berichte (zu zitieren als Chem. Ber.) setzen die Berichte der Deutschen Chemischen Gesellschaft (zu zitieren als Ber. Dtsch. Chem. Ges.) fort.

2. Die „Berichte“ enthalten Originalmitteilungen aus allen Gebieten der Chemie.

3. Die „Berichte“ erscheinen monatlich; ein Registerheft beschließt jeden Band.

4. Die Verantwortung für ihre Mitteilungen tragen die Verfasser selbst.

5. Es werden grundsätzlich nur Arbeiten aufgenommen, die vorher weder im Inland noch im Ausland veröffentlicht worden sind.

6. Eine Anweisung zur Abfassung von Manuskripten für die „Berichte“ wird auf Wunsch zugestellt.

7. Manuskripte sind zu senden an Redaktion der Chemischen Berichte. Dr. H. Zahn, Am Klopferspitz, D-8033 Martinsried. Telefon (089) 8585830.

8. Der Eingang der Abhandlungen wird den Autoren am Tage der Registrierung angezeigt.

9. Es werden nur Manuskripte in deutscher oder englischer Sprache aufgenommen. Allen Beiträgen ist eine knappe Zusammenfassung (summary) in beiden Sprachen voranzustellen, einschließlich Titel in der jeweils zweiten Sprache.

10. Der Autor muß das alleinige Urheberrecht besitzen. Mit der Annahme des Manuskriptes durch die Redaktion überträgt er dem Verlag Chemie das ausschließliche Nutzungsrecht, insbesondere das Recht der Vervielfältigung wie Fotokopie, Mikrofilm - oder mit irgendeinem anderen Verfahren - oder das Manuskript in eine von Maschinen, insbesondere von Datenverarbeitungsmaschinen, verwendbare Sprache zu übergeben oder zu übersetzen (auch in fremde Sprachen).

11. Den Autoren werden 75 Sonderdrucke unentgeltlich portofrei zugesandt. Wünscht ein Autor mehr als 75 Abzüge, so ist dies auf dem Manuskript oder spätestens bei Rücksendung der Korrektur auf dieser zu vermerken. Den Autoren werden nur die Selbstkosten für die Zahl der die Freiexemplare überschreitenden Sonderabzüge berechnet.

12. Anfragen nach dem Verbleib nicht eingetroffener Berichte-Hefte oder Sonderdrucke sind zu richten an: Verlag Chemie GmbH, Pappelallee 3, Postfach 1260/1280, D-6940 Weinheim, Telefon (06201) 602-1.

Die Chemischen Berichte erscheinen monatlich. Der Jahresbezugspreis beträgt 670. - DM zuzügl. Versandgebühren. Einzelheft 76. - DM. In diesem Preis ist die Mehrwertsteuer enthalten. Die Bezugsbedingungen für Mitglieder der Gesellschaft Deutscher Chemiker werden auf Anfrage von der Geschäftsstelle, Carl-Bosch-Haus, Varrentrappstraße 40-42, Post fach 900440, D-6000 Frankfurt 90, mitgeteilt. - Bestellungen richten Sie bitte an Ihre Fachbuchhandlung oder unmittelbar an den Verlag. - Abbestellungen nur bis spätestens 2 Monate vor Ablauf des Kalenderjahres. - Die Lieferung erfolgt auf Rechnung und Gefahr des Empfängers. Gerichtsstand und Erfüllungsort: Weinheim/Bergstr.

Verlag und Anzeigenabteilung: Verlag Chemie GmbH, Pappelallee 3, Post fach 1260/1280, D-6940 Weinheim. Fernsprecher (06201) 602-1, Fernschreiber 465516 vchwh d.

For USA and Canada

Published monthly by Verlag Chemie GmbH, Weinheim, Federal Republic of Germany. Annual subscription price: $\$ 455.00$ including postage. Second-class postage paid at Jamaica, N. Y. Printed in the Federal Republic of Germany. Air-freighted and mailed in the United States by Publication Expediting Inc., 200 Meacham Avenue, Elmont, N. Y. 11003 . Subscribers in North America should place their order through Verlag Chemie International Inc., Plaza Centre, Suite E, 1020 N. W. Sixth Street, Deerfield Beach, Florida 33441. 
114. Jahrgang 1981

\section{ANORGANISCHE CHEMIE}

Werner Helmut und Hofmann Werner: Basische Metalle, XXX: Zur Reaktivität und Dynamik der Komplexe $\mathrm{C}_{5} \mathrm{H}_{5} \mathrm{Co}\left(\mathrm{PR}_{3}\right)_{2}(\mathrm{R}=\mathrm{Me}, \mathrm{Et}),\left(\mathrm{C}_{5} \mathrm{H}_{4} \mathrm{R}\right) \mathrm{Co}\left(\mathrm{PMe}_{3}\right)_{2}$ und $\left(\mathrm{C}_{5} \mathrm{H}_{3} \mathrm{RR}^{\prime}\right) \mathrm{Co}\left(\mathrm{PMe}_{3}\right)_{2}$ : Einfluß sterischer Faktoren auf die Lewis-Basizität elek-

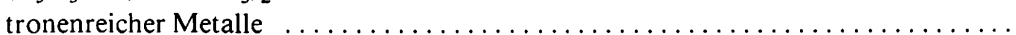

Wieghardt Karl, Holzbach Wolfgang, Hofer Edgar und Weiss Johannes: Synthesen mit (Hydroxylamido(1-)-O,N)molybdän(VI)-Komplexen und Alkylcyaniden. Die Strukturen von $\operatorname{Bis}\left(N\right.$-hydroxy- $N$-methylacetamidinato $\left.(1-)-O, N^{\prime}\right)(N$-methylhydroxylamido$(1-)-O, N)$ oxomolybdän(VI)-perchlorat und von $\mu$-Oxo-bis $[(N$-hydroxy- $N$-methylacetamidinato(1-)-O, $N^{\prime} \rightarrow M o^{1} ; O \rightarrow M o^{2}$ )-cis-dioxo]dimolybdän(VI) $\ldots \ldots \ldots \ldots$

Anton Klaus, Fußstetter Hermann und Nöth Heinrich: Beiträge zur Chemie des Bors, 118: Struktur und Dynamik von Hexamethylborazin-Aluminiumbromid .........

Köpf Hartmut und Voigtländer Rolf: Anionoide Polyphosphane als Chelatliganden: Triphosphanato- $P^{1}, P^{3}$-Komplexe des Titanocen-, Zirconocen- und Hafnocen-

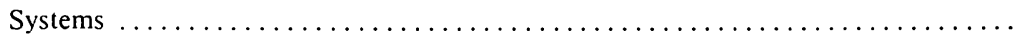

Kunze Udo, Bolz Paul-Reiner und Winter Werner: Nucleophile Addition von Triorganozinn-Anionen an Kohlenstoffdisulfid. Röntgenstrukturbestimmung von Methyl-

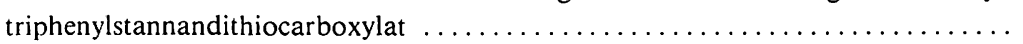

Berke Heinz, Bankhardt Willi, Huttner Gottfried, Seyerl v. Joachim und Zsolnai Laszlo: Eisenkomplexe als Modellverbindungen zur homogenen Hydrierung von Kohlenmonoxid

\section{ORGANISCHE CHEMIE}

Dyong Ingolf, Friege Hannelore, Luftmann Heinrich und Merten Hans: Synthesen biologisch wichtiger Kohlenhydrate, 27: Totalsynthese und Konfigurationsbestimmung $\mathrm{Me}-\stackrel{\mathrm{C}}{\mathrm{I}}^{3}$-NHR-verzweigter 2,3,6-Tridesoxyhexosen

Goerdeler Joachim, Tiedt Marie-Louise und Nandi Kumaresh: Über Thioacyl-isocyanate, $\mathrm{XV}$ : Reaktionen mit nucleophilen C-Verbindungen $\ldots \ldots \ldots \ldots \ldots \ldots \ldots \ldots$

Rüchardt Christoph, Golzke Volker und Range Günter: Organische Peroxide, XIV: Der thermische Zerfall polycyclischer Brückenkopf-Peroxycarbonsäureester $\ldots \ldots \ldots \ldots$.

Hoffmann Reinhard W., Ladner Wolfgang, Steinbach Klaus, Massa Werner, Schmidt Roland und Snatzke Günther: Stereoselektive Synthese von Alkoholen, IX: Absolute Konfiguration von Stegobinon . . . . . . . . . . . . . . . . . . . .

Hoffmann Reinhard W. und Helbig Wilfried: Stereoselektive Synthese von Alkoholen, X: Diastereoselektive Synthese von ( - )- $\delta$-Multistriatin 
Franke Wilfried, Schwarz Helmut, Thies Helga, Chandrasekhar Jayaraman, Schleyer Ragué von Paul, Hehre Warren J., Saunders Martin und Walker Gary: Entartete Isomerisierung via Kohlenstoff-Platzwechsel beim Cyclopentyl-Kation in der Gasphase. Experimenteller und theoretischer Nachweis der Existenz eines pyramidalen $\mathrm{C}_{5} \mathrm{H}_{9}^{+}$-Kations bei der unimolekularen Ethylen-Eliminierung $\ldots \ldots \ldots \ldots \ldots \ldots$

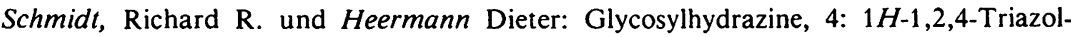
nucleoside - Synthese von Virazol . ..........................

Jung Karl-Heinz, Schmidt Richard R. und Heermann Dieter: Glycosylhydrazine, 5: Aufbauende Synthese der 5'-Säureamid-Derivate von Pyrazol-, Pyrazolo[3,4- $d$ ]pyrimidin- und ${ }^{1} H$-1,2,4-Triazol-nucleosiden . . . . . . . . . . . . . . . . . . . .

Kaupp Gerd und Grüter Heinz-Willi: Bekannte und neue Reaktionstypen bei der Photoreaktion von Stilbenen mit cyclischen Iminen $\ldots \ldots \ldots \ldots \ldots \ldots \ldots \ldots \ldots$

Giese Bernd und Mazumdar Pronab: Synthese von Allylthioethern $\ldots \ldots \ldots \ldots \ldots \ldots$

Gompper Rudolf und Vogt Hans-Hubert: Das Allopolarisierungsprinzip und seine Anwendungen, IV: Substituenteneffekte bei der Methylierung von Enolat-Anionen ....

Vogt Hans-Hubert und Gompper Rudolf: Das Allopolarisierungsprinzip und seine Anwendungen, V: ${ }^{13} \mathrm{C}$-NMR-Spektren von Enolaten und verwandten Carbanionen ....

Hoffmann H. M. R. und Vathke-Ernst Heidrun: Cycloadditionen von Allyl-Kationen, 29: Säurekatalysierte Reaktionen von 2,3-Dimethyl-3-penten-2-ol mit Cyclopentadien in zwei Phasen. Bildung von sieben- und fünfgliedrigen Ringen $\ldots \ldots \ldots \ldots \ldots$

Langhals Heinz: Die quantitative Beschreibung der Lösungsmittelpolarität binärer Gemische unter Berücksichtigung verschiedener Polaritätsskalen $\ldots \ldots \ldots \ldots \ldots \ldots$

Koßmehl Gerhard und Nuck Rolf: Wittig-Reaktionen mit Cyanhydrinen sowie Redoxreaktion des 4-Nitromandelsäurenitrils $\ldots \ldots \ldots \ldots \ldots \ldots \ldots \ldots \ldots \ldots \ldots$

Roedig Alfred und Fleischmann Klaus: Thermolyse von 5-chlorsubstituierten $(2 Z, 4 Z)$ 2,4-Pentadienthiosäure- $S$-alkylestern zu $2 H$-Pyran-2-thionen und $2 H$-Thiopyran-

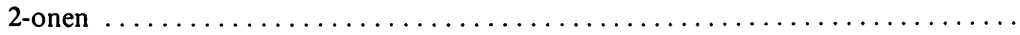

Braun Manfred und Esdar Michael: Umsetzung von Aldehyden mit Enolaten des Dithian-

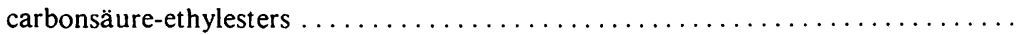




\section{INORGANIC CHEMISTRY}

Werner Helmut and Hofmann Werner: Basic Metals, XXX: Reactivity and Dynamics of the Complexes $\mathrm{C}_{5} \mathrm{H}_{5} \mathrm{Co}\left(\mathrm{PR}_{3}\right)_{2}(\mathrm{R}=\mathrm{Me}, \mathrm{Et}),\left(\mathrm{C}_{5} \mathrm{H}_{4} \mathrm{R}\right) \mathrm{Co}\left(\mathrm{PMe}_{3}\right)_{2}$, and $\left(\mathrm{C}_{5} \mathrm{H}_{3} \mathrm{RR}^{\prime}\right) \mathrm{Co}$ $\left(\mathrm{PMe}_{3}\right)_{2}$ : Influence of Steric Effects on the Lewis Basicity of Electron-rich Metals ...

Wieghardt Karl, Holzbach Wolfgang, Hofer Edgar, and Weiss Johannes: Syntheses with (Hydroxylamido(1 -)-O,N)-molybdenum(VI) Complexes and Alkyl Cyanides. The Structures of $\operatorname{Bis}\left(N\right.$-hydroxy- $N$-methylacetamidinato $\left.(1-)-O, N^{\prime}\right)(N$-methylhydroxylamido(1 - )- $O, N$ )oxomolybdenum(VI) Perchlorate and of $\mu$-Oxo-bis $[(N$-hydroxy- $N$ methylacetamidinato(1-)- $O, N^{\prime} \rightarrow M o^{1} ; O \rightarrow M o^{2}$ )-cis-dioxo]dimolybdenum(VI) ..

Anton Klaus, Fußstetter Hermann, and Nöth Heinrich: Contributions to the Chemistry of Boron, 118: Structure and Dynamics of Hexamethylborazine-Aluminium Tribromide .

Köpf Hartmut and Voigtländer Rolf: Anionoidal Polyphosphanes as Chelate Ligands: Triphosphanato- $P^{1}, P^{3}$ Complexes of the Titanocene, Zirconocene, and Hafnocene

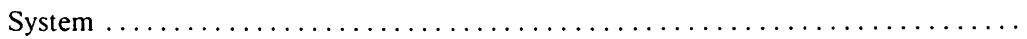

Kunze Udo, Bolz Paul-Reiner, and Winter Werner: Nucleophilic Addition of Triorganotin Anions to Carben Disulfide. $X$-Ray Structure Determination of Methyl Tri-

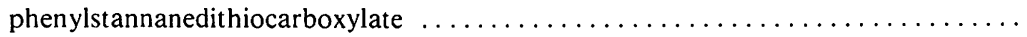

Berke Heinz, Bankhardt Willi, Huttner Gott fried, Seyerl v. Joachim, and Zsolnai Laszlo: Iron Complexes as Model Compounds for the Homogeneous Hydrogenation of Carbon Monoxide

\section{ORGANIC CHEMISTRY}

Dyong Ingolf, Friege Hannelore, Luftmann Heinrich, and Merten Hans: Syntheses of Biologically Important Carbohydrates, 27: Total Synthesis and Determination of the Configuration of $\mathrm{Me}-\stackrel{1}{\mathrm{C}}^{3}-\mathrm{NHR}-\mathrm{Branched}$ 2,3,6-Trideoxy Hexoses $\ldots \ldots \ldots \ldots$

Goerdeler Joachim, Tiedt Marie-Louise, and Nandi Kumaresh: Thioacyl Isocyanates, XV: Reaction with Nucleophilic C-Compounds $\ldots \ldots \ldots \ldots \ldots \ldots \ldots \ldots$

Rüchardt Christoph, Golzke Volker, and Range Günter: Organic Peroxides, XIV: The Thermal Decomposition of Polycyclic tert-Butyl Bridgehead Peroxycarboxylates ...

Hoffmann Reinhard W., Ladner Wolfgang, Steinbach Klaus, Masa Werner, Schmidt Roland, and Snatzke Günther: Stereoselective Synthesis of Alcohols, IX: Absolute Configuration of Stegobinone

Hoffmann Reinhard W. and Helbig Wilfried: Stereoselective Synthesis of Alcohols, X: Diastereoselective Synthesis of $(-)-\delta$-Multistriatin $\ldots \ldots \ldots \ldots \ldots \ldots \ldots \ldots$

Franke Wilfried, Schwarz Helmut, Thies Helga, Chandrasekhar Jayaraman, Schleyer Ragué von Paul, Hehre Warren J., Saunders Martin, and Walker Gary: Degenerate Carbon Skeleton Isomerization of the Cyclopentyl Cation in the Gas Phase. Experimental and Theoretical Evidence for the Existence of a Pyramidal $\mathrm{C}_{5} \mathrm{H}_{9}^{+}$Cation as an Intermediate in the Unimoleculare Ethylene Elimination $\ldots \ldots \ldots \ldots \ldots$ 
Schmidt Richard R. and Heermann Dieter: Glycosylhydrazines, 4: 1H-1,2,4-Triazole Nucleosides - Synthesis of Virazole . . . . . . . . . . . . . . . . . . .

Jung Karl-Heinz, Schmidt Richard R., and Heermann Dieter: Glycosylhydrazines, 5: Synthesis of Pyrazole-, Pyrazolo[3,4- $d$ ] pyrimidine-, and $1 H-1,2,4$-Triazole Nucleoside-5'-amide Derivatives via Riburonamide Hydrazones . . . . . . . . . . . . .

Kaupp Gerd and Grüter Heinz-Willi: Known and New Types of Reactions in the Photoreaction of Stilbenes with Cyclic Imines $\ldots \ldots \ldots \ldots \ldots \ldots \ldots \ldots \ldots \ldots . \ldots \ldots . \ldots \ldots$

Giese Bernd and Mazumdar Pronab: Synthesis of Allyl Thioethers $\ldots \ldots \ldots \ldots \ldots \ldots \quad 2859$

Gompper Rudolf and Vogt Hans-Hubert: The Allopolarization Principle and its Applications, IV: Substituent Effects in the Methylation of Enolate Anions ......... 2866

Vogt Hans-Hubert and Gompper Rudolf: The Allopolarization Principle and its Applications, $\mathrm{V}:{ }^{13} \mathrm{C}$ NMR Spectra of Enolates and Related Carbanions ..............

Hoffmann H. M. R. and Vathke-Ernst Heidrun: Cycloadditions of Allyl Cations, 29: Acid Catalyzed Reactions of 2,3-Dimethyl-3-penten-2-ol and Cyclopentadiene in Two Phases. Formation of Seven- and Five-membered Rings . . . . . . . . . . . . .

Langhals Heinz: A Quantitative Description of Polarity of Binary Solvent Mixtures Using Different Polarity Scales ....................................

Koßmehl Gerhard and Nuck Rolf: Wittig Reactions of Cyanohydrines and Redox Reaction of 4-Nitromandelonitrile ...............................

Roedig Alfred and Fleischmann Klaus: Thermolysis of 5-Chloro-substituted S-Alkyl (2Z,4Z)-2,4-Pentadienethioates to $2 H$-Pyran-2-thiones and $2 H$-Thiopyran-2-ones ..

Braun Manfred and Esdar Michael: Reaction of Aldehydes with Enolates of Ethyl Dithianecarboxylate 
Anton, K., Fußstetter, $H$. und

Nöth, $H$. 2723

Bankhardt, $W$. s. Berke, $H$. 2754

Berke, H., Bankhardt, W., Huttner, G.,

Seyerl, v., J. und Zsolnai, L. . ... 2754

Bolz, P.-R. s. Kunze, U. .......... 2744

Braun, M. und Esdar, M. . . . . . . . 2924

Chandrasekhar, J. s. Franke, W. . . . 2808

Dyong, I., Friege, H., Luftmann, $H$. und Merten, $H$. 2669

Esdar, M. s. Braun, M. 2924

Fleischmann, K. s. Roedig, A. 2921

Franke, W., Schwarz, H., Thies, $H$. , Chandrasekhar, J., Schleyer, Ragué, von P.; Hehre, W. J., Saunders, $M$. und Walker, $G$ 2808

Friege, $H$. s. Dyong, $I$. 2669

Fußstetter, H. s. Anton, $K$. 2723

Giese, B. und Mazumdar, $P$. 2859

Goerdeler, J., Tiedt, M.-L. und

Nandi, $K$. 2713

Golzke, V. s. Rüchardt, $C$. 2769

Gompper, R. und Vogt, H.-H. 2866

- s. Vogt, H.-H. 2884

Grüter, H.-W. s. Kaupp, G. 2844

Heermann, D. s. Jung, K.-H. 2834

- s. Schmidt, R. R. 2825

Hehre, W. J. s. Franke, $W$. 2808

Helbig, W. s. Hoffmann, R. W.

Hofer, E. s. Wieghardt, $K$. 2802

Hoffmann, H. M. R. und

Vathke-Ernst, $H$. 2898

Hoffmann, R. W. und Helbig, W. 2802

-, Ladner, W., Steinbach, K., Massa, W., Schmidt, $R$. und Snatzke, $G$.
Hofmann, W. s. Werner, H. . . . . . 2681

Holzbach, W. s. Wieghardt, K. ..... 2700

Huttner, G. s. Berke, H. . . ........ 2754

Jung, K.-H., Schmidt, R. R. und

Heermann, D. . . . . . . . . . 2834

Kaupp, G. und Grüter, H.-W. . ..... 2844

Köpf, $H$. und Voigtländer, $R$. . . . . 2731

Koßmehl, G. und Nuck, R. . . . . . . 2914

Kunze, U., Bolz, P.-R. und

Winter, $W . \ldots \ldots \ldots \ldots \ldots \ldots 2744$

Ladner, W. s. Hoffmann, R. W. . . . 2786

Langhals, H. . . . . . . . . . . . . . 2907

Luftmann, H. s. Dyong, I. . . . . . . . 2669

Massa, W. s. Hoffmann, R. W. . . . . 2786

Mazumdar, P. s. Giese, B. . . . . . . . 2859

Merten, H. s. Dyong, I . . . . . . . . . . 2669

Nandi, K. s. Goerdeler, J. . . . . . . . . 2713

Nöth, H. s. Anton, K. . .......... 2723

Nuck, R. s. Koßmehl, G. . . . . . . ... 2914

Range, G. s. Rüchardt, C. . . . . . . . 2769

Roedig, A. und Fleischmann, K. .... 2921

Rüchardt, C., Golzke, $V$. und

Range, C. ................. 2769

Saunders, M. s. Francke, W. . . . . . . . 2808

Schleyer, Ragué, von, P. s. Franke, W. . 2808

Schmidt, Richard $R$. und

Heermann, D. ............ 2825

- s. Jung, K.-H. ............. 2834

Schmidt, Roland s. Hoffmann, R. W. .. 2786

Schwarz, H. s. Franke, W. . . . . . . 2808

Seyerl, v., J. s. Berke, H. . . . . . . . . 2754

Snatzke, G. s. Hoffmann, R. W. . ... 2786

Steinbach, K. s. Hoffmann, R. W. . . . 2786

Thies, H. s. Franke, W. . . . . . . . 2808

Tiedt, M.-L. s. Goerdeler, J. . . . . . . 2713

2786 Vathke-Ernst, H. s. Hoffmann, H. M. R. 2898 
Vogt, H.-H. und Gompper, $R$.

2884 Werner, $H$. und Hofmann, $W$.

2681

- s. Gompper, $R$.

Voigtländer, $R$. s. Köpf, $H$. 2731

Wieghardt, K., Holzbach, W., Hofer, E. und Weiss, J. ............ 2700

Walker, G. s. Franke, W. 2808

Vinter, W. s. Kunze, U. . . . . .... 2744

Weiss, J. s. Wieghardt, $K$.

2700 Zsolnai, L. s. Berke, $H$. 2754 


\title{
Die quantitative Beschreibung der Lösungsmittelpolarität binärer Gemische unter Berücksichtigung verschiedener Polaritätsskalen
}

\author{
Heinz Langhals \\ Chemisches Laboratorium der Universität Freiburg, \\ Albertstr. 21, D-7800 Freiburg i. Br.
}

Eingegangen am 23. Januar 1981

Die Lösungsmittelpolaritäten von 12 binären Gemischen sind als Funktion ihrer Zusammensetzung unter Berücksichtigung von 6 Polaritätsskalen untersucht worden. Sie erfüllen alle quantitativ eine Zwei-Parameter-Gleichung, mit deren Hilfe u. a. Abweichungen von der linearen Korrelation der Polaritätsskalen bei Gemischen erklärt werden können.

\section{A Quantitative Description of Polarity of Binary Solvent Mixtures Using Different Polarity Scales}

The solvent polarities of 12 binary mixtures have been examined using 6 polarity scales as a function of their composition. They are quantitatively described by a simple, closed form, twoparameter equation which can explain e.g. deviations from linear correlations of polarity scales for mixtures.

Die quantitative Beschreibung der Polarität eines Lösungsmittels ist ein wichtiges Problem in der Organischen Chemie. Da rein physikalische Solvens-Eigenschaften für die Beschreibung der makroskopischen Lösungsmittelpolarität nur verhältnismäßig grobe Maße darstellen ${ }^{1-3)}$, brachte die Entwicklung von empirischen Polaritätsskalen auf der Basis lösungsmittelabhängiger Prozesse einen wesentlichen Fortschritt ${ }^{4-9}$ ). Die für reine Lösungsmittel beobachtete lineare Korrelation dieser Skalen 6, 12,20-22) legt zwar die Existenz eines allgemein gültigen Polaritätsmaßes nahe, demgegenüber werden jedoch bei Lösungsmittelgemischen größere Abweichungen beobachtet $1,5,11$ ).

In vorangegangenen Arbeiten 23 -26) konnte gezeigt werden, daß die Polarität eines binären Gemisches, bestimmt nach der $E_{\mathrm{T}}(30)$-Skala von Dimroth, Reichardt und Mitarbb. ${ }^{10)}$, als Funktion seiner Zusammensetzung durch Gleichung (1) beschrieben wird $\left(P_{\mathrm{G}}=E_{\mathrm{T}}(30)\right)$.

$$
P_{\mathrm{G}}=E_{\mathrm{D}} \cdot \ln \left(\frac{c_{\mathrm{p}}}{c^{*}}+1\right)+P_{\mathrm{G}}^{\circ}
$$

$c_{\mathrm{p}}$ in $\mathrm{Gl}$. (1) ist die Konzentration der polareren Komponente (die Komponente mit dem größeren $E_{\mathrm{T}}(30)$-Wert) und $P_{\mathrm{G}}^{\circ}$ der $P_{\mathrm{G}}$-Wert der reinen schwächer polaren Komponente. Die Größen $E_{\mathrm{D}}$ (Energiedurchgriff) und $c^{*}$ (Erscheinungskonzentration) sind empirisch zu bestimmende $\mathrm{Pa}$ rameter der Gleichung ${ }^{23)}$. Die $E_{\mathrm{T}}$ (30)-Werte [vgl. Gl. (1)] sind die molaren Anregungsenergien der Solvatochromiebande des Pyridiniophenolats 1 und werden aus $\lambda_{\max }$ dieser Bande mit Hilfe von Gl. (2) berechnet.

$$
E_{\mathrm{T}}(30)\left[\mathrm{kcal} \cdot \mathrm{mol}^{-1}\right]=28590\left[\mathrm{kcal} \cdot \mathrm{nm} \cdot \mathrm{mol}^{-1}\right] / \lambda_{\max }
$$

Chem. Ber. 114 (1981)

(C) Verlag Chemie GmbH, D-6940 Weinheim, 1981

$0009-2940 / 81 / 0808-2907 \$ 02.50 / 0$ 
Im vorliegenden Beitrag wird geprüft, ob Gl. (1) auch andere Polaritätsskalen für binäre Gemische quantitativ beschreibt. Besonderer Wert wurde dabei auf die Polaritätsskalen $1-4$ gelegt, da sie häufig verwendet werden und auf unterschiedlichen Effekten beruhen. Skala 1 (bzw. 6) basiert auf der lösungsmittelabhängigen Lage der $\pi \rightarrow \pi^{*}$-Solvatochromiebande von 1 (bzw. 6), einer negativ solvatochromen Substanz, Skala 4 auf einer analogen, jedoch positiven solvatochromen Verschiebung der Absorption. Skala 3 verwendet als Meßsonde die lösungsmittelabhängige Lage eines Charge-Transfer-Übergangs und Skala 2 die Solvolysereaktion von tert-Butylchlorid. Zu beachten ist weiterhin, daß Skala 5 nach Untersuchungen von Dimroth, Reichardt und Mitarbb. empfindlich auf Wasserstoffbrücken bildende H-Donatoren anspricht ${ }^{10)}$ und für protische Lösungsmittel keine gute Korrelation mit den anderen Polaritätsskalen ergibt. Dieser Effekt ist in abgeschwächter Form ebenfalls für 1 in Erwägung gezogen worden 15,21). Bei Skala 2 wird diskutiert, ob die Abhängigkeit der Geschwindigkeitskonstante der Solvolyse von 2 vom umgebenden Medium auf einer Solvatation des Übergangszustandes der Reaktion oder eher des Grundzustands beruht $9,27-30$ ).

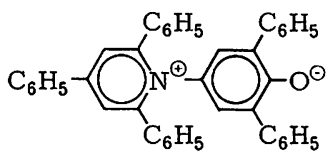

$1 E_{\mathrm{T}}(30)^{6,10,11)}$<smiles>CC(C)(C)Cl</smiles>

$2 Y^{9)}$

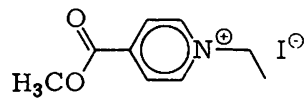

$3 Z^{12)}$

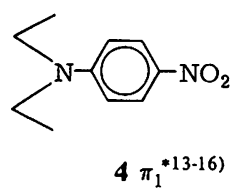

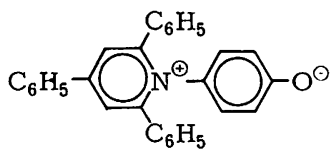

$5 E_{\mathrm{T}}(1)^{10)}$

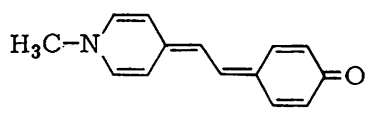

6 MOED ${ }^{17 \cdot 19)}$

\section{Ergebnisse}

Die Polaritäten der Gemische von Tab. 1 wurden nach den beschriebenen Polaritätsskalen bestimmt. Die untersuchten Polaritätsskalen erfüllen für alle Lösungsmittelgemische von Tab. 1 die Gleichung (1).

Beim Auftragen von $P_{\mathrm{G}}$ (und davon abgeleitet $\left.P_{\mathrm{G}}-P_{\mathrm{G}}^{\circ}\right)$ gegen $\ln \left(c_{\mathrm{p}} / c^{*}+1\right)$ werden Geraden erhalten, von denen einige in Abb. 1 wiedergegeben sind.

Wie sich auch leicht visuell feststellen läßt, ist Gl. (1) über mehrere Zehnerpotenzen von $c_{\mathrm{p}}$ erfüllt. Die Abweichungen von den Geraden sind rein statistisch und liegen in der gleichen Größenordnung wie die Unsicherheiten der jeweiligen Meßmethode. Die Ergebnisse der Messungen sind in Tab. 1 zusammengestellt. Wie bereits erwähnt, spricht die Solvatochromie von 5 stark auf Wasserstoffbrücken-bildende Substanzen $a^{10)}$. Umso bemerkenswerter ist, daß Gl. (1) bei Skala 5 sogar für das stark zu HBrücken neigende Gemisch Wasser/Ethanol (Tab. 1) erfüllt ist.

Bei den Gemischen Wasser/Ethanol wird außerdem eine Besonderheit beobachtet, über die bei anderen Gemischen bereits berichtet worden ist ${ }^{26)}$. Für WasserKonzentrationen $c_{\mathrm{p}}>30 \mathrm{~mol} \cdot \mathrm{1}^{-1}\left(c_{\mathrm{k}}\right)$ geht die Funktion nach Gl. (1) in eine weitere mit anderen Parametern $E_{\mathrm{D}}$ und $c^{*}$ über (Bildung von 2 Geraden. Für $c_{\mathrm{p}}>c_{\mathrm{k}}$ und $c_{\mathrm{p}}<$ $c_{\mathrm{k}}$ gilt jeweils Gl. (1), aber mit unterschiedlichen Parametern). Diese besondere Eigenschaft des Gemisches Wasser/Ethanol tritt bei allen untersuchten Polaritätsskalen auf 
Tab. 1. Vergleich der Polaritätsskalen

\begin{tabular}{|c|c|c|c|c|c|c|c|c|}
\hline Skala a) & Gemisch b) & $c_{\mathrm{p}}{ }^{\mathrm{c}, \mathrm{d})}$ & $E_{\mathrm{D}}^{\mathrm{e}, \mathrm{I})}$ & $c^{* \mathrm{~d}, \mathrm{~g})}$ & $r^{\text {h) }}$ & $n^{i)}$ & $\sigma_{E_{\mathrm{D}}}^{\mathrm{e}, \mathrm{j})}$ & $\left.P_{\mathrm{G}}^{\mathrm{o}} \mathrm{e}, \mathrm{k}\right)$ \\
\hline$E_{\mathrm{T}}(30)$ & Wasser/Ethanol ${ }^{\circ}$ ) & $0.06-38.7$ & 1.64 & 6.83 & 0.99232 & 27 & 0.04 & 51.9 \\
\hline$E_{\mathrm{T}}(30)$ & Methanol/Aceton & $0.03-25$ & 2.53 & 0.10 & 0.99973 & 29 & 0.02 & 42.2 \\
\hline $\begin{array}{l}Y \\
Y \\
Y\end{array}$ & $\begin{array}{l}\text { Wasser/Ethanol }{ }^{\text {l) }} \\
\text { (Wasser/Ethanol) }{ }^{1,0, p)} \\
\text { Wasser/Methanol }{ }^{1)}\end{array}$ & $\begin{array}{l}0.06-30 \\
30-55.4 \\
0.0-55.4\end{array}$ & $\begin{array}{l}0.64 \mathrm{~m}, \mathrm{n}) \\
0.85^{\mathrm{m}, \mathrm{q})} \\
3.91 \mathrm{~m}, \mathrm{~s})\end{array}$ & $\begin{array}{l}7.48 \\
(0.09) \\
24.0\end{array}$ & $\begin{array}{l}0.99956 \\
(0.99625) \\
0.99972\end{array}$ & $\begin{array}{c}12 \\
(17) \\
11\end{array}$ & $\begin{array}{l}0.01 \mathrm{~m}) \\
(0.02)^{\mathrm{m})} \\
0.03^{\mathrm{m})}\end{array}$ & $\begin{array}{l}-2.05^{\mathrm{m})} \\
(-5.92)^{\mathrm{m})} \\
-1.12\end{array}$ \\
\hline$Z$ & Methanol/Dioxan & $0.03-24.7$ & 8.52 & 2.66 & 0.99384 & 31 & 0.18 & 63.0 \\
\hline$Z$ & Methanol/Aceton & $0.03-24.7$ & 4.66 & 0.65 & 0.99993 & 31 & 0.01 & 66.3 \\
\hline$Z$ & Ethanol/Acetonitril r) & $0.02-17.1$ & 2.76 & 0.80 & 0.99781 & 47 & 0.04 & 72.2 \\
\hline$\pi_{1}^{*}$ & Wasser/Ethanol o) & $0.06-55.4$ & -1.71 & 3.08 & -0.99846 & 31 & 0.02 & 74.8 \\
\hline$\pi_{1}^{*}$ & Ethanol/n-Heptan & $0.02-17.1$ & -3.13 & 2.93 & -0.99681 & 33 & 0.04 & 78.7 \\
\hline $\begin{array}{l}E_{\mathrm{T}}(1) \\
E_{\mathrm{T}}(1)\end{array}$ & $\begin{array}{l}\text { Wasser/Ethanolo) } \\
(\text { Wasser/Ethanol) }\end{array}$ & $\begin{array}{l}0.06-30 \\
30-55.4\end{array}$ & $\begin{array}{c}6.67 \\
(11.37)\end{array}$ & $\begin{array}{l}49.7 \\
0.01\end{array}$ & $\begin{array}{l}0.99740 \\
(0.98809)\end{array}$ & $\begin{array}{l}34 \\
(7)\end{array}$ & $\begin{array}{l}0.10 \\
(0.79)\end{array}$ & $\begin{array}{l}35.2 \\
(11.3)\end{array}$ \\
\hline MOED & Wasser/Ethanol $\left.{ }^{\circ}\right)$ & $0.06-55.4$ & 2.04 & 5.47 & 0.99168 & 24 & 0.06 & 55.9 \\
\hline$M O E D$ & Methanol/Aceton & $0.03-24.7$ & 2.25 & 0.31 & 0.99952 & 30 & 0.01 & 49.3 \\
\hline
\end{tabular}

a) Polaritätsskala, siehe Text. - b) Untersuchtes Lösungsmittelgemisch, die polarere Komponente ist zuerst aufgeführt. - c) Untersuchter Konzentrationsbereich. - d) In mol $\cdot 1^{-1}$. - e) In $\mathrm{kcal} \cdot \mathrm{mol}^{-1}$, zum Umrechnen in SI-Einheiten wird mit dem Faktor 4.2 multipliziert. - f) Energiedurchgriff nach Gl. (1). - g) Erscheinungskonzentration nach Gl. (1). - h) Korrelationskoeffizient nach Gl. (1). - i) Anzahl der Meßwerte. - j) Varianz von $E_{\mathrm{D}}$. k) Polaritätswert der reinen, unpolareren Komponente. - l) Unter Verwendung der Werte nach Lit. ${ }^{8}$ ). - m) In $Y$-Einheiten, zur Umrechnung in kcal · $\mathrm{mol}^{-1}$ muß mit einem Faktor 0.733 multipliziert werden. - n) $3.70 \mathrm{kcal} \cdot \mathrm{mol}^{-1}$. - o) Für $c_{\mathrm{p}}>30 \mathrm{~mol} \cdot \mathrm{l}^{-1}\left(c_{\mathrm{k}}\right)$ wird eine $\mathrm{zweite}$, steilere Gerade beobachtet. - p) $c_{\mathrm{p}}>c_{\mathrm{k}}$. - q) $4.94 \mathrm{kcal} \cdot \mathrm{mol}^{-1}$. - r) Für $c_{\mathrm{p}}>6.8 \mathrm{~mol} \cdot \mathrm{1}^{-1}$ wird eine zweite Gerade nach Gl. (1) mit geringerer Steigung beobachtet. s) $2.87 \mathrm{kcal} / \mathrm{mol}$. 
und ist für einige Beispiele aus Abb. 2 zu ersehen. Der Übergang bei $c_{k}$ erfolgt sehr abrupt und stellt einen Knick in der Funktion nach Gl. (1) (bzw. $P_{\mathrm{G}}=\mathrm{f}\left(\ln c_{\mathrm{p}}\right)^{23)}$ ) dar. Diese Besonderheit im polaren Verhalten des Gemisches verdient insofern Beachtung, als häufig im Gemisch Wasser/Ethanol lösungsmittelabhängige Effekte untersucht werden, und weil nach den gefundenen Ergebnissen für $c_{\mathrm{p}}>c_{\mathrm{k}}$ und $c_{\mathrm{p}}<c_{\mathrm{k}}$ unterschiedliche Lösungsmittelstrukturen zu erwarten stehen.

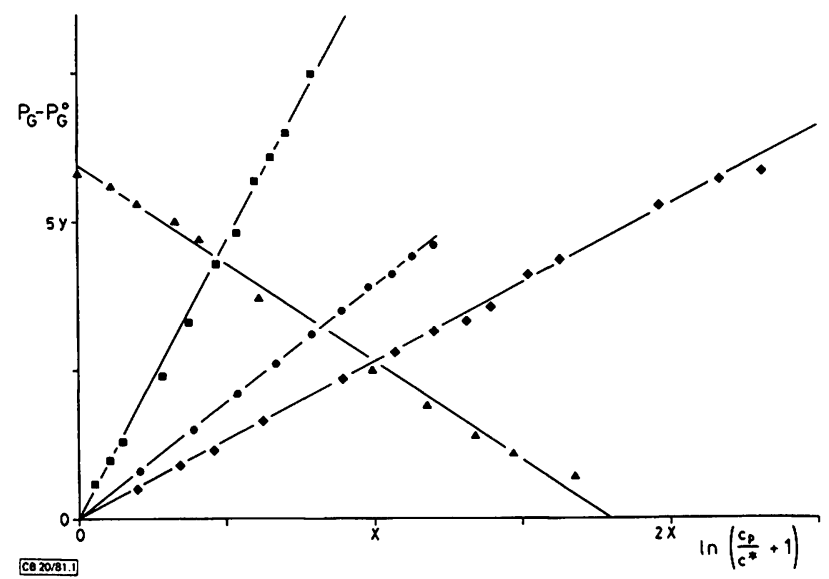

Abb. 1. Linearer Zusammenhang zwischen $P_{\mathrm{G}}$ und $\ln \left(c_{\mathrm{p}} / c^{*}+1\right)$ für verschiedene Polaritätsskalen [Gl. (1)]

$E_{\mathrm{T}}$ (30) (1) Methanol/Aceton, $\mathrm{x}=2, \mathrm{y}=2 ; \bullet: Y(2)$ Wasser/Methanol, $\mathrm{x}=1, \mathrm{y}=1$; $\mathbf{a}: Z$

(3) Methanol/Aceton, $\mathrm{x}=2, \mathrm{y}=1 ; \boldsymbol{\Delta}: \pi_{1}^{*}(4)$ Ethanol/n-Heptan, $\mathrm{x}=1, \mathrm{y}=1$; Ordinate: $P_{\mathrm{G}}-P_{\mathrm{G}}^{\circ}+5.9$

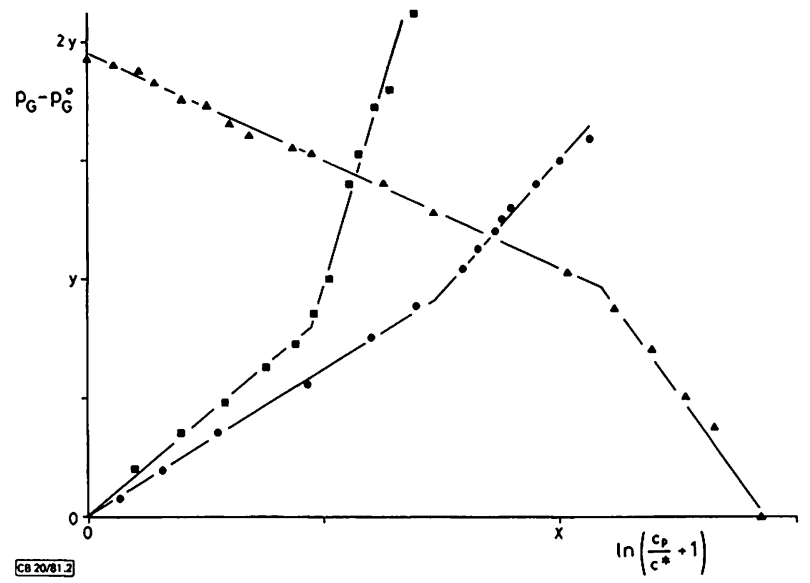

Abb. 2. Doppelgeraden nach Gl. (1) für das Gemisch Wasser/Ethanol

-: $Y(2), \mathrm{x}=2, \mathrm{y}=1 ; \boldsymbol{\nabla}: E_{\mathrm{T}}(\mathbf{1})(5), \mathrm{x}=1, \mathrm{y}=4 ; \Delta: \pi_{1}^{*}(4), \mathrm{x}=2, \mathrm{y}=4$;

Ordinate: $P_{\mathrm{G}}-P_{\mathrm{G}}^{\mathrm{o}}+7.7$

Chem. Ber. $114(1981)$ 


\section{Diskussion der Ergebnisse}

Wie aus den geschilderten Ergebnissen zu ersehen ist, beschreibt Gl. (1) das polare Verhalten binärer Gemische bei Polaritätsskalen, die aus sehr unterschiedlichen Effekten abgeleitet worden sind. Die Vielzahl und Verschiedenartigkeit der untersuchten binären Lösungsmittelgemische (s.a. Lit. ${ }^{26,31)}$ ) sowie der Polaritätsskalen (Tab. 1) legt nahe, daß Gl. (1) eine weitgehend gültige Beschreibung des polaren Verhaltens von binären Flüssigkeitsgemischen darstellt und läßt verstehen, daß im allgemeinen bei Auftragen der Polarität eines Gemisches nicht, wie zunächst erwartet, Geraden, sondern gekrümmte Kurven ${ }^{5,10,16,22,32)}$ erhalten werden.

Die Gültigkeit von Gl. (1) ist ein weiterer Hinweis auf die Existenz eines allgemein gültigen Polaritätsmaßes: es steht zu erwarten, daß den zunächst empirisch aufgestellten Polaritätsskalen gleichartige Ursachen zugrunde liegen. Die beobachteten Gesetzmäßigkeiten ermöglichen z. B. die Polarität eines Gemisches exakt einzustellen und sind z. B. bei Solvolysereaktionen zu berücksichtigen, wenn im Verlaufe der Reaktion die Konzentration $c^{*}$ einer Komponente über- oder unterschritten wird. Die Polarität des Mediums kann sich dann stark verändern. Weiterhin können unter Anwendung von Gl. (1) die Eigenschaften von Polymeren durch Copolymerisation oder durch Zusätze (Weichmacher) gezielt beeinflußt werden. Hierüber wird in anderem Zusammenhang berichtet. Bemerkenswert ist, wie schon gesagt, daß Skala 5, die stark von Wasserstoffbrückenbildnern beeinflußt wird, ebenfalls Gl. (1) erfüllt. Dieses Ergebnis ist ein Indiz dafür, daß Polaritätseffekte, die auf Wasserstoffbrückenbindungen zurückgeführt werden, zusammen mit anderen Effekten einheitlich beschrieben werden können.

Bisher ungeklärt war die Frage, weshalb bei binären Lösungsmittelgemischen Abweichungen von der linearen Korrelation der Polaritätsskalen auftreten, während sie für reine Lösungsmittel gut erfüllt ist. Dieser unerwartete und bislang nicht verstandene Befund findet nun auf der Basis des vorgestellten quantitativen Zusammenhangs [Gl. (1)] eine befriedigende Erklärung: Wie schon anderen Orts dargelegt ${ }^{23)}$, läßt sich die Funktion nach Gl. (1) in zwei Bereiche unterteilen. Für Konzentrationen $c_{\mathrm{p}} \ll c^{*}$ verhalten sich die Beiträge beider Lösungsmittel zur Gesamtpolarität additiv, die Funktion nach Gl. (1) kann also durch eine lineare Funktion angenähert werden. Für $c_{\mathrm{p}} \gg c^{*}$ ist diese Funktion jedoch logarithmisch. Der experimentell zugängliche Bereich $c_{\mathrm{p}}$ reicht nur von $c_{\mathrm{p}}=0$ bis zur Konzentration des reinen, polareren Lösungsmittels ( $\left.c_{\mathrm{p}} \max \right)$. Gilt $c_{\mathrm{p}} \max \ll c^{*}$, so verhalten sich die Polaritäten der Lösungsmittelkomponenten näherungsweise additiv. In diesem Falle ist eine Korrelation der Polaritätsskalen zu erwarten. Kann jedoch experimentell $c^{*}$ deutlich überschritten werden $\left(c_{\mathrm{p}} \max >c^{*}\right)$, gilt für $c_{\mathrm{p}}>c^{*}$ der genannte lineare Zusammenhang nicht mehr (Krümmung). Der dann gültige, nichtlineare (transzendent irrationale) Zusammenhang sollte sich bei verschiedenen Polaritätsskalen unterschiedlich stark auswirken, da beide Parameter von Gl. (1) zum Tragen kommen. Eine lineare Beziehung zwischen den Polaritätsskalen ist somit nicht mehr gegeben. Die beobachteten Abweichungen sind also eine notwendige Folge der Gültigkeit von Gl. (1).

Herrn Prof. Dr. Christoph Rüchardt sei an dieser Stelle für seine Unterstützung und der Deutschen Forschungsgemeinschaft für die Förderung dieser Arbeit gedankt. 


\section{Experimenteller Teil}

UV-Spektren: DMR 21 der Fa. Zeiss.

4-(2,4,6-Triphenyl-1-pyridinio)phenolat $\left(E_{\Upsilon}(1), 5\right)^{10)}$ und 1-(Diethylamino)-4-nitrobenzol ( $\pi_{1}^{*}$, 4) ${ }^{33,34)}$ werden nach Lit.-Angaben synthetisiert und die verwendeten Lösungsmittel nach üblichen Methoden gereinigt.

2,6-Diphenyl-4-(2,4,6-triphenyl-1-pyridinio)phenolat $\left(E_{\mathrm{T}}(30), 1\right)$ : Die Synthese erfolgt nach Lit.-Angaben ${ }^{10)}$. Abweichend von diesen wird 1 aus $450 \mathrm{ml}$ Methanol/Wasser (1:1), bezogen auf $1.0 \mathrm{~g}$ Ausgangsmaterial (1-Perchlorat), umkristallisiert.

1-Ethyl-4-(methoxycarbonyl)pyridinium-iodid $(Z, 3)^{12)}: 20 \mathrm{ml}$ Ethyliodid werden unter Umschwenken mit $3.0 \mathrm{~g}$ (19.6 mmol) 4-Pyridincarbonsäure-methylester unter Eiskühlung versetzt. Die Mischung färbt sich rot, und es fallen orangefarbene Kristalle aus. Man läßt über Nacht bei $5^{\circ} \mathrm{C}$ unter Feuchtigkeitsausschluß stehen. Das auskristallisierte 3 wird abgesaugt und mit Ether gewaschen (große, orangefarbene Kristalltafeln). 3 kann ohne weitere Reinigung für die spektroskopischen Untersuchungen eingesetzt werden. Ausb. $5.6 \mathrm{~g}(92 \%)$. Schmp. $111-114^{\circ} \mathrm{C}$ (Lit. ${ }^{12)}$ $\left.111-112^{\circ} \mathrm{C}\right)$.

1,4-Dimethylpyridinium-iodid 17-19): Zu $20 \mathrm{ml}$ Methyliodid werden unter Eiskühlung und Umschwenken nach und nach $2.0 \mathrm{~g}(21.5 \mathrm{mmol})$ 4-Methylpyridin gegeben. Die Mischung färbt sich unter leichtem Erwärmen gelb, und es fällt ein farbloser, kristalliner Niederschlag aus. Zur Vervollständigung der Kristallisation läßt man über Nacht bei $-20^{\circ} \mathrm{C}$ stehen, saugt anschließend ab und wäscht mit Ether. Die isolierte Verbindung ist lichtempfindlich und wird ohne weitere Reinigung umgesetzt. Ausb. $4.25 \mathrm{~g}\left(84 \%\right.$ ), Schmp. $144^{\circ} \mathrm{C}$ (Lit. $\left.{ }^{19)} 144^{\circ} \mathrm{C}\right)$.

4-[2-(1-Methyl-4(1 H)-pyridinyliden)ethyliden]-2,5-cyclohexadien-1-on $\left.\quad(M O E D, \quad 6)^{17-19}\right)$ : $4.25 \mathrm{~g}$ (18.1 mmol) 1,4-Dimethylpyridinium-iodid, $1.5 \mathrm{ml}$ Piperidin und $2.18 \mathrm{~g}(17.9 \mathrm{mmol}) 4$ Hydroxybenzaldehyd werden in $20 \mathrm{ml}$ absol. Ethanol suspendiert und anschließend $4 \mathrm{~h}$ unter Rückfluß gekocht. Die beim Abkühlen auf Raumtemp. ausgefallenen Kristalle werden abgesaugt, mit wenig absol. Ethanol gewaschen und in $100 \mathrm{ml} 0.2 \mathrm{M} \mathrm{KOH}$ suspendiert. Die Mischung wird 30 min lang auf $80^{\circ} \mathrm{C}$ erwärmt und heiß filtriert. Beim Abkühlen fallen große, rotviolette Kristallplatten aus, die ohne weitere Reinigung für die spektroskopischen Messungen verwendet werden können. Ausb. $3.2 \mathrm{~g} \mathrm{(85 \% ).} \mathrm{Schmp.} 220^{\circ} \mathrm{C}$ (Lit. ${ }^{19)} 220^{\circ} \mathrm{C}$ ).

Bestimmung von $E_{\mathrm{D}}$ und $c^{*}$ binärer Lösungsmittelgemische: Die Bestimmung der Parameter von Gl. (2) erfolgt analog Lit.-Angaben 23, 24). Die Auswertung der Meßergebnisse ist mit dem Rechenprogramm POLAR ${ }^{23)}$ (in FORTRAN V) am Rechenzentrum der Universität Freiburg vorgenommen worden.

1) A. Streitwieser jr., Chem. Rev. 56, 620 (1956).

2) $K$. Schwetlick, Kinetische Methoden zur Untersuchung von Reaktionsmechanismen, 1. Aufl., S. 143 - 174, VEB, Berlin 1971.

3) S. Brownstein, Can. J. Chem. 38, 1590 (1960).

4) S. Winstein, E. Grunwald und H. W. Jones, J. Am. Chem. Soc. 73, 2700 (1951).

5) C. Reichardt, Solvent Effects in Organic Chemistry, 1. Aufl., Verlag Chemie, Weinheim 1979.

6) C. Reichardt, Angew. Chem. 91, 119 (1979); Angew. Chem., Int. Ed. Engl. 18, 98 (1979).

7) A. Koppel und V. A. Palm, in N. B. Chapman und J. Shorter, Advances in Linear Free Energy Relationships, 1. Aufl., S. 203-280, Plenum Press, London 1973.

8) A. H. Fainberg und S. Winstein, J. Am. Chem. Soc. 78, 2770 (1956).

9) S. Winstein und A. H. Fainberg, J. Am. Chem. Soc. 79, 5937 (1957).

10) K. Dimroth, C. Reichardt, T. Siepmann und F. Bohlmann, Liebigs Ann. Chem. 661, 1 (1963).

11) $K$. Dimroth und C. Reichardt, Fortschr. Chem. Forsch. 11, 1 (1968).

12) E. M. Kosower, J. Am. Chem. Soc. 80, 3253 (1958). 
13) M. J. Kamlet, E. G. Kayser, J. W. Eastes und W. H. Gilligan, J. Am. Chem. Soc. 95, 5210 (1973).

14) M. J. Kamlet und $R$. W. Taft, J. Am. Chem. Soc. 98, 377 (1976).

15) M. J. Kamlet, J. L. Abboud und R. W. Taft, J. Am. Chem. Soc. 99, 6027 (1977).

16) M. J. Kamlet, T. N. Hall, J. Boykin und R. W. Taft, J. Org. Chem. 44, 2599 (1979).

17) L. G. S. Brooker, G. H. Keyes und D. W. Heseltine, J. Am. Chem. Soc. 73, 5350 (1951).

18) S. Hünig und $O$. Rosenthal, Liebigs Ann. Chem. 592, 161 (1955).

19) M. J. Minch und S. S. Shah, J. Chem. Educ. 54, 709 (1976).

20) T. E. Gough, D. E. Irish und I. R. Lantzke, Spectroscopic Measurements, in A. K. Covington und T. Dickinson, Physical Chemistry of Organic Solvent Systems, S. $405 \mathrm{ff}$., Plenum Press, London 1973.

21) F. W. Fowler, A. R. Katritzki und R. J. D. Rutherford, J. Chem. Soc. B 1971, 460.

22) $R$. D. Cramer, J. Am. Chem. Soc. 102, 1849 (1980).

23) $H$. Langhals, Nouv. J. Chim. 5, 97 (1981).

24) $H$. Langhals, Z. Anal. Chem. 305, 26 (1981).

25) $H$. Langhals, DOS 3043897.2 vom 21.11.1980.

26) $H$. Langhals, DOS 3043984.0 vom 21.11.1980.

27) E. M. Arnett, W. G. Bentrude und P. MC C. Duggleby, J. Am. Chem. Soc. 87, 2048 (1965).

28) A. J. Parker, U. Mayer, R. Schmid und V. Gutmann, J. Org. Chem. 43, 1843 (1978).

29) M. H. Abraham, J. Chem. Soc., Perkin Trans. 2 1972, 1343.

30) M. H. Abraham und R. J. Abraham, J. Chem. Soc., Perkin Trans. 2 1974, 47.

31) $H$. Langhals, Z. Anal. Chem., im Druck.

32) H. Langhals, E. Fritz und I. Mergelsberg, Chem. Ber. 113, 3662 (1980).

33) G. M. Bennett und E. V. Bell, Org. Synth., Coll. Vol. II, 223 (1950).

34) W. C. Davies, Bull. Soc. Chim. Fr. 1932, 295. 